\title{
An $\alpha$-quaternary chiral latam derivative, YH-304 as a novel broad-spectrum anticancer agent
}

\author{
SU JUNG HWANG ${ }^{1,2}$, HYEUNG-GEUN PARK ${ }^{3}$, YOHAN PARK $^{1,2}$ and HYO-JONG LEE ${ }^{1,2}$ \\ ${ }^{1}$ College of Pharmacy and Inje Institute of Pharmaceutical Sciences and Research, and \\ ${ }^{2} \mathrm{u}$-Healthcare and Anti-aging Reearch Center (u-HARC), Inje University, Gimhae, Gyeongnam 621-749; ${ }^{3}$ Research Institute \\ of Pharmaceutical Science and College of Pharmacy, Seoul National University, Seoul 151-742, Republic of Korea
}

Received June 27, 2016; Accepted September 30, 2016

DOI: $10.3892 /$ ijo.2016.3726

\begin{abstract}
Previously, we reported that $\alpha$-quaternary chiral lactam derivatives have broad spectrum anticancer activity. However, the underlying molecular mechanisms and its relevance are largely unknown. In the present study, we report progress on $\alpha$-quaternary chiral lactam analogues that address this, focusing on the novel analogue $\mathrm{YH}-304$ as a candidate to broadly target human cancer cells. The effect of YH-304 on cell transformation was assessed by clonogenic assay in non-small cell lung cancer cells (NSCLCs) A549 and 226B. Proapoptotic activity of YH-304 was determined by TUNEL assay and cleaved PARP, cleaved caspase-9, and Bax as markers for apoptosis. The p53-dependency and therapeutic spectrum of YH-304 was assessed by western blot analysis, real-time PCR, and cell viability assays in cells expressing endogenous wild or mutant p53. The effect of $\mathrm{YH}-304$ on angiogenesis in vivo was examined by bFGF-mediated angiogenesis assay in zebrafish. Finally, the effect of YH-304 on AKT and ERK activation (phosphorylation) as a putative mechanism underlying the effect of YH-304 on bFGF-mediated angiogenesis was assessed using western blotting. We found that YH-304 significantly decreases the colony-forming activities of both A549 and 226B cells, inducing cellular apoptosis. Unlike nutlin-3 (p53 pathway activator), YH-304 did not affect the expression levels of p53 and its target gene such as p21 and thus showed p53-independent anticancer activity with broad spectrum. In addition, YH-304 inhibited bFGF-induced angiogenesis in vivo through mediating AKT and ERK signaling pathway, which plays an important role in bFGF activation and angiogenesis. Taken together, our data indicate that YH-304
\end{abstract}

Correspondence to: Dr Yohan Park or Professor Hyo-Jong Lee, College of Pharmacy and Inje Institute of Pharmaceutical Sciences and Research, Inje University, 197 Inje-ro, Gimhae, Gyungnam 621-749, Republic of Korea

E-mail: yohanpark@inje.ac.kr

E-mail: hjlee@inje.ac.kr

Key words: $\alpha$-quaternary chiral lactam, angiogenesis, p53, AKT, ERK may represent a novel therapeutic option for the treatment of cancer in a p53-independent manner.

\section{Introduction}

Cancer is a world-wide disease urgently requiring novel therapeutic agents having a new class of structure (1-3). An oxindole structure possessing a bicyclic aromatic heterocycle with a benzene ring fused to $\gamma$-lactam is one of the structural classes (4). Diverse derivatives based on oxindole have been reported for development of new type anticancer candidates (5-8). Representative natural product possessing oxindole is spirotryprostatin A, which is well known for its anticancer activities through blockade of the G2/M progression in mouse mammary carcinoma cells $(4,9)$. The other oxindole small molecule, sunitinib was developed as a multi-targeted receptor tyrosine kinase inhibitor and has been widely used in the treatment of renal cell carcinoma and imatinib-resistant gastrointestinal stromal tumors (10,11). In 2005, Ding et al (13) developed the MDM2 inhibitor from spiro-oxindole structure of spirotryprostatin $\mathrm{A}$ and the $\mathrm{IC}_{50}$ value of $\mathrm{MDM} 2$ inhibitor was $0.83 \mu \mathrm{M}$ in LNCaP prostate cancer cells (12-14). In a previous study, we developed a series of oxindole derivatives having simplified $\alpha$-quaternary chiral lactam and evaluated their anticancer efficacy $(2,15)$. However, the mechanisms of action underlying its anticancer effects are largely unknown and need to be better understood to allow this therapy to be used for clinical trials. This prompted us to study further the anti-tumorigenic effects of $\mathrm{YH}-304$, including its underlying molecular mechanisms.

The p53 tumor suppressor mediates growth arrest, senescence, and apoptosis in response to a broad array of cellular damage and protects the organism against the propagation of cells with damaged DNA (16). In unstressed cells, MDM2 acts as an p53-antagonist by inducing ubiquitination of p53, which results in p53 degradation (17). Therefore, inhibition of the p53-MDM2 interaction using chemical or biological inhibitors becomes an attractive strategy for cancer therapy (18). Many MDM2 inhibitors have been developed and evaluated in a large panel of tumors, including lymphomas $(19,20)$. Nutlin-3 is one of the specific inhibitors of the MDM2-p53 interaction and leads to stabilization and activation of the p53 $(21,22)$. However, recently, several studies reported the limitation 
of the efficacy of nutlin-3 such as resistance formation (23). Therefore, there is an urgent need to develop novel MDM2 inhibitors or p53 activators.

Zebrafish has become a widely used model in pre-clinical drug screening and the mechanistic studies of angiogenesis, since its embryo is small and optically transparent to visualize the whole vessels $(24,25)$. Especially, the use of vascular specific transgene allows for the detection of individual growing cells or vessel formation (26). To date, many types of cancer models have been developed in zebrafish and providing excellent tools for anticancer drug discovery through candidate drug testing $(27,28)$. In the present study, we used transgenic fluorescent zebrafish $\operatorname{tg}(f i 1: E G F P)$ to evaluate the anti-angiogenic efficacy of YH-304 in live zebrafish embryos.

In the present study, we showed the anti-tumorigenic effect of YH-304 using in vitro clonogenic assay, TUNEL assay, and cell viability assay, as well as the effect on bFGF-induced in vivo angiogenesis. At the molecular level, we found that YH-304 increases the number of TUNEL-positive cells and the expression level of cleaved PARP, cleaved caspase- 9 and Bax, resulting in apoptotic cell death. YH-304 did not alter the p53 or its target gene expression levels. In addition, YH-304 potently inhibited angiogenesis of bFGF-mediated AKT and ERK phosphorylation. The anti-tumorigenic and anti-angiogenic potential of YH-304 to induce apoptosis with broad spectrum and downregulate ERK/AKT activity suggests a putative use in anticancer therapy.

\section{Materials and methods}

Cell cultures. HeLa (human cervical carcinoma cell line), HCT-116 (human colon carcinoma cell line), A549, 226B (human non-small lung cancer cell lines), and MDA-MB-231 (human breast cancer cell line) were purchased from the American Type Culture Collection (ATCC; Manassas, VA, USA) and maintained at $37^{\circ} \mathrm{C}$ in a humidified $5 \% \mathrm{CO}_{2}$ atmosphere. HeLa, HCT-116 and MDA-MB-231 cells were cultured in Dulbecco's modified Eagle's medium and A549 and 226B cells were cultured in RPMI-1640 medium supplemented with $10 \%$ fetal bovine serum (FBS) and 1\% antibiotics (Invitrogen, Carlsbad, CA, USA).

Reagents. Recombinant bFGF was purchased from BD Biosciences (Franklin Lakes, NJ, USA), whereas the antiGAPDH, $\alpha$-Tubulin, PARP and p53 antibodies were purchased from Santa Cruz Biotechnology (Santa Cruz, CA, USA) and the anti-Akt, p-Akt, cleaved caspase-9, Bax, ERK and p-ERK antibodies used were purchased from Cell Signaling Technology (Boston, MA, USA). Nutlin-3 was purchased from Sigma-Aldrich (St. Louis, MO, USA). YH-304 [(S)Methyl 1-benzhydryl-3-(4-methylbenzyl)-2-oxopiperidine3 -carboxylate] was produced as previously described (2).

Clonogenic assay. In brief, A549 and 226B cells were seeded at $2 \times 10^{3}$ cells/well on a 6 -well plate and allowed to attach overnight. The next day, medium was replaced with fresh medium containing different concentrations $(0.5$ and $2 \mu \mathrm{M})$ of YH-304 and incubated for $2 \mathrm{~h}$. Following incubation, cells were harvested by trypsinization and counted. For clonogenic assay, $4 \mathrm{ml}$ of cell suspension $(50$ cells $/ \mathrm{ml})$ was seeded in a 6-well plate and were incubated at $37^{\circ} \mathrm{C}$ for 14 days without any disturbance. Following incubation, the medium was removed and colonies were fixed and stained with $0.01 \%(\mathrm{w} / \mathrm{v})$ crystal violet for $30 \mathrm{~min}$. A colony consisting of at least 50 cells was counted with ImageJ software (NIH, Bethesda, MD, USA). The experiment was carried out twice in triplicate.

Western blot analysis. Western blot analyses were performed as previously described using the antibodies mentioned above. The respective protein bands were detected by chemiluminescence (FUSION SL4; Vilber Lourmat, Marne la Vallée, France).

Real-time PCR analysis. Total RNA was isolated from cells using TRIzol ${ }^{\circledR}$ reagent (Invitrogen) and transcribed using a PrimeScript First Strand cDNA Synthesis kit (Takara, Shiga, Japan) according to the manufacturers' protocols. Quantitative real-time PCR was performed in triplicate on Rotor-Gene ${ }^{\circledR} \mathrm{Q}$ (Roche Diagnostics, Indianapolis, IN, USA) using LightCycler ${ }^{\circledR}$ SYBR-Green I Master (Roche Diagnostics), and data were analyzed on the basis of threshold cycle values of each sample and normalized with $\beta$-actin. The PCR primers used in this study are listed below and were purchased from Bioneer: forward p53 5'-GCCCAACAACACCAGCTCCT-3' and reverse p53 5'-GCCCAACAACACCAGCTCCT-3'; forward p21 5'-GGCCTCCTGACCCACAGCAG-3' and reverse p21 5'-GGGCTCAACTCAACACCCACC-3'; forward $\beta$-actin 5'-AGAGGGAAATCGTGC-3' and reverse $\beta$-actin 5'-GGC CGTCAGGCAGCTCATAG-3'.

Cell death assays by TUNEL staining. DNA strand breaks during apoptosis were detected by TUNEL of free 3'-OH ends of cleaved DNA. Following YH-304 treatment for 24 h, 226B cells were fixed in $4 \%(\mathrm{v} / \mathrm{v})$ paraformaldehyde for $30 \mathrm{~min}$ on ice. Fixed cells were washed once in phosphate-buffered saline (PBS) and incubated for $60 \mathrm{~min}$ at $37^{\circ} \mathrm{C}$ in $50 \mu \mathrm{l}$ of TdT-containing solution (Roche Diagnostics, Mannheim, Germany). Following TUNEL staining, all samples were washed once in PBS and nuclei were stained using 4'-6-Diamidino-2phenylindole (DAPI; Invitrogen). Images were obtained with an Axiovert M200 microscope (Carl Zeiss AG, Oberkochen, Germany).

Cell viability assay. HCT-116, HeLa, A549 and MDA-MB-231 cells were treated with different concentration of YH-304 as indicated. After $24 \mathrm{~h}, 20 \mu \mathrm{l}$ of CellTiter $96^{\circledR}$ Aqueous One solution reagent (Promega, Madison, WI, USA) was added and then read at $490 \mathrm{~nm}$. Dose-response curves were plotted to determine half-maximal inhibitory concentrations $\left(\mathrm{IC}_{50}\right)$ for the compounds with SigmaPlot software.

Zebrafish angiogenesis assay. Fertilized zebrafish (Danio rerio) eggs of the transgenic strain expressing green fluorescent protein (GFP) under the flk-1 promoter $(f k-1: G F P)$ were used for in vivo imaging of embryonic vascular development. At $24 \mathrm{~h}$ post-fertilization (hpf), EGFP-expressing $\operatorname{tg}($ flil:EGFP) embryos were manually dechorionated and anesthetized with $0.003 \%$ tricaine. Anesthetized embryos were then transferred onto a $4 \%$ agarose gel for microinjection. Approximately $5 \mathrm{nl}$ of Matrigel containing either PBS or basic fibroblast growth 

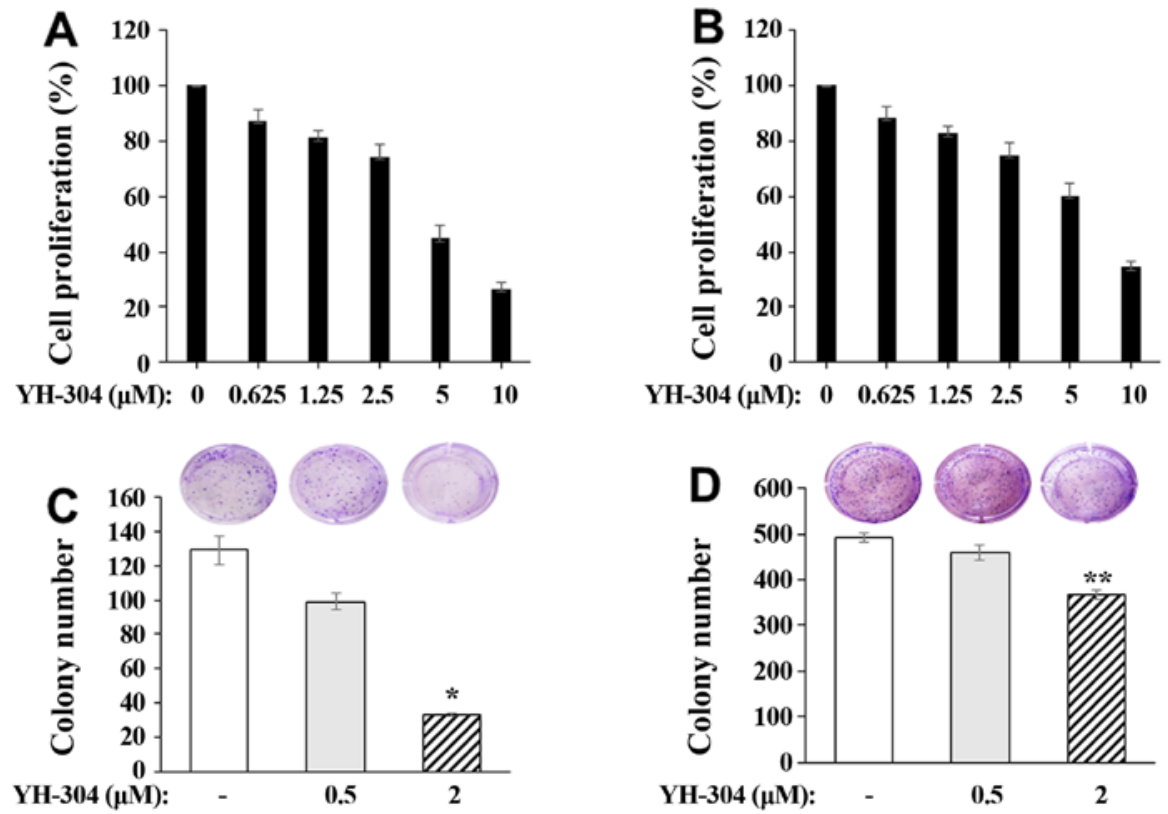

Figure 1. YH-304 inhibits proliferation and colony formation of A549 and 226B NSCLCs. (A and B) After incubation with YH-304 ranging from 0.625 to $10 \mu \mathrm{M}$ for $24 \mathrm{~h}$, MTT was added and the absorbance was measured at $570 \mathrm{~nm}$ in an ELISA reader. (A) Effect of YH-304 on cellular proliferation in A549 cells. (B) Effect of YH-304 on cellular proliferation in 226B cells. (C and D) Cells were pretreated with 0.5 or $2 \mu \mathrm{M}$ of YH-304 and then were seeded (2,000 cells/well) in triplicate onto 6-well plates and treated with 0.5 or $2 \mu \mathrm{M}$ of YH-304. After 2 weeks, the colonies formed were stained using crystal violet and counted. (C) Colony formation by YH-304 in A549 cells. (D) Colony formation by YH-304 in 226B cells. Values are expressed relative to vehicle-treated cells, normalized to $100 \% \pm$ standard deviation (SD), and were obtained from three independent experiments. ${ }^{*} \mathrm{P}<0.05,{ }^{* *} \mathrm{P}<0.01$.
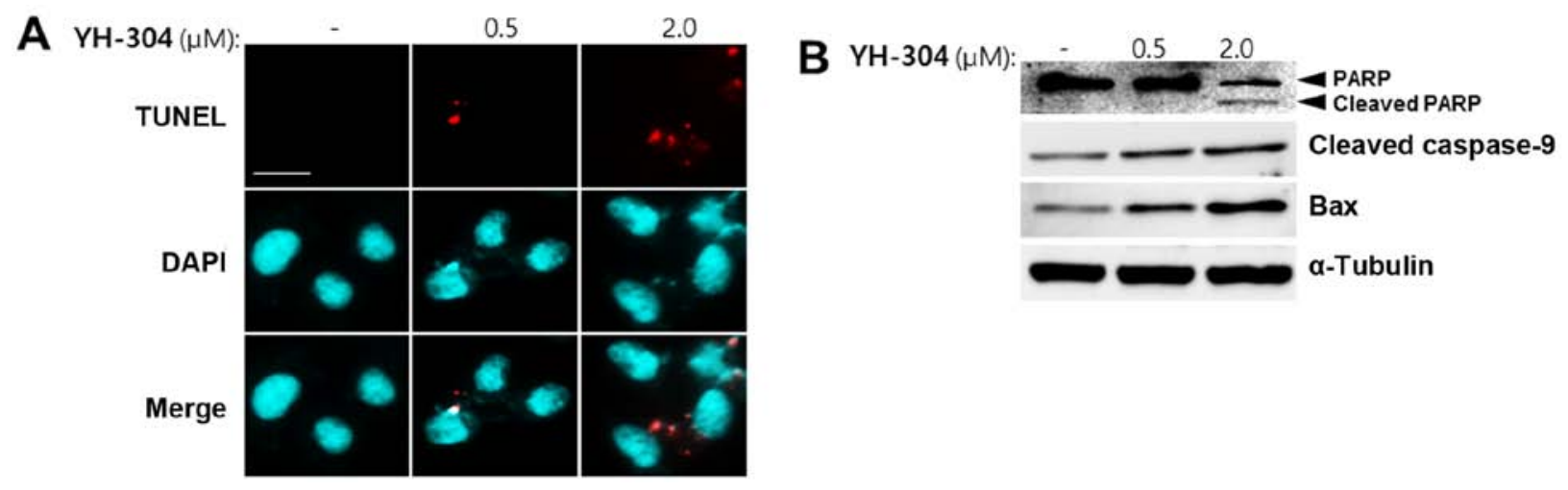

Figure 2. YH-304 induces apoptosis of A549 cells. (A) The TUNEL assay of 0.5 or $2 \mu \mathrm{M}$ YH-304-treated A549 cells. Apoptotic cell death was evaluated by TUNEL staining (red, $\mathrm{x} 40$ objective lens) after 24-h treatment of YH-304. Representative images of three independent experiments show the effect of YH-304 on TUNEL-positive programmed cell death. Scale bar is $10 \mu \mathrm{m}$. (B) Effect of YH-304 on PARP cleavage, cleaved caspase-9 and Bax in A549 cells. A549 cells were treated with YH-304 ranging from 0.5 to $2 \mu \mathrm{M}$ for $24 \mathrm{~h}$. Western blotting was performed to evaluate the cleaved PARP, intact PARP, cleaved caspase-9, Bax and $\alpha$-tubulin expression levels. $\alpha$-tubulin was used as an internal control.

factor (bFGF, $25 \mathrm{ng} / \mathrm{ml}$ ) was injected into the yolk sac of each embryo using microinjector (Narishige International, Inc., East Meadow, NY, USA). Non-filamentous borosilicate glass capillary needles were used for the microinjection $(0.75 \mathrm{~mm}$ internal- and $1.0 \mathrm{~mm}$ external-diameter). Embryos were transferred into housing-keeping water immediately after injection and incubated with $0.003 \%$ 1-phenyl-2-thiourea (Sigma-Aldrich) at $28.5^{\circ} \mathrm{C}$ to prevent pigmentation. YH-304 treatment was carried out immediately after Matrigel injection and embryos were examined for angiogenesis using a fluorescent microscope (Carl Zeiss) $24 \mathrm{~h}$ later.

Statistical analysis. All values are expressed as means \pm SE. Statistical significance was set at $\mathrm{P}<0.05 ; \mathrm{P}<0.05 ; \mathrm{P}<0.001$.

\section{Results}

YH-304 inhibits proliferation and colony formation of A549 and 226B NSCLC cells. To investigate the effect of YH-304 on the cancer cell proliferation, A549 and 226B NSCLC cells were pretreated with or without $\mathrm{YH}-304$ ranging from 0.625 to $10 \mu \mathrm{M}$ for $24 \mathrm{~h}$ and then measured by 3-[4,5-methylthiazol2-yl]-2,5-diphenyl-tetrazolium bromide (MTT) assay. We found that $\mathrm{YH}-304$ inhibited cancer cell proliferation in both A549 (Fig. 1A) and 226B (Fig. 1B) NSCLC cells in a dose-dependent manner. The anticancer effect of YH-304 was subsequently confirmed by clonogenic assay in A549 and 226B NSCLC cells. As observed above, we found that YH-304 suppresses colony formation ability of both A549 (Fig. 1C) 

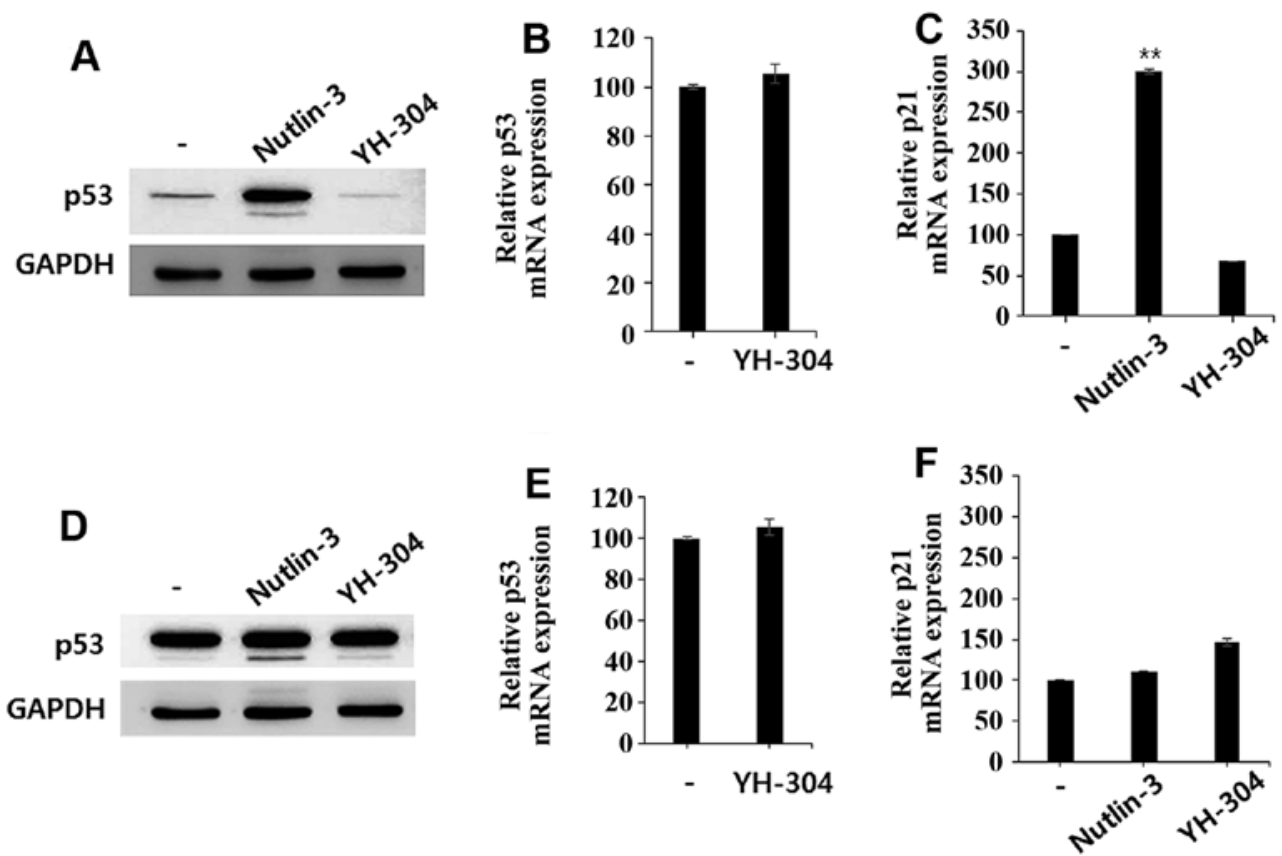

Figure 3. YH-304 did not affect p53 expression levels in HCT-116 and MDA-MB-231 cells. Cells were treated with either $10 \mu \mathrm{M}$ nutlin-3 (a well-known MDM2 antagonist) or $10 \mu \mathrm{M}$ of YH-304 for $24 \mathrm{~h}$. (A) Effect of p53 protein expression in HCT-116 (expressing wild-type p53) cells. (B) Effect of p21 mRNA expression in HCT-116 cells. (C) Effect of p53 protein expression in MDA-MB-231 (expressing mutant-type p53) cells. (D) Effect of p21 mRNA expression in MDA-MB-231 cells. (E) Effect of YH-304 on p53 mRNA expression in HCT-116 cells. (F) Effect of YH-304 on p53 mRNA expression in MDA-MB-231 cells. GAPDH and $\beta$-actin were used as internal controls.

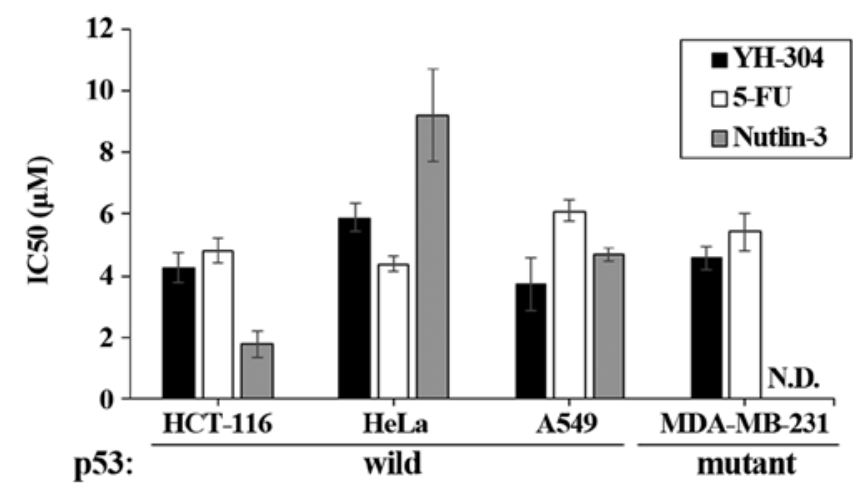

Figure 4. YH-304 shows broad spectrum anticancer activity via a p53-independent manner. The p53-independent effectiveness of $\mathrm{YH}-304$ against four different types of cancer cells. The average $\mathrm{IC}_{50}$ values of $\mathrm{YH}-304,5-\mathrm{FU}$ (5-fluorouracil), and nutlin-3 (a well-known MDM2 inhibitor) were analyzed against three cancer cell lines (HCT-116, HeLa and A549) expressing wild-type p53 and MDA-MB-231 expressing mutant-type p53. Cells were treated with YH-304 ranging from 0.039 to $10 \mu \mathrm{M}$ for $24 \mathrm{~h}$. Cells treated with DMSO (equivalent volume) were used as vehicle control. N.D., not determined.

and 226B (Fig. 1D) NSCLC cells. This suggests that YH-304 has potential for anticancer efficacy in NSCLC cells.

YH-304 induces apoptosis of A549 NSCLC cells. We next set out to determine whether YH-304 is involved in apoptosis using TUNEL assay. When A549 cells were treated with 0.5 or $2.0 \mu \mathrm{M}$ YH-304, TUNEL-positive cells were increased as measured by fluorescence microscopy at individual cell level (Fig. 2A). From these cell images, it is clear that the red fluorescence intensity of individual cells increased in a concentration-dependent manner indicating apoptosis by YH-304. Additionally, we evaluated whether YH-304 can induce apoptotic cell death using apoptotic markers such as cleaved poly(ADP-ribose) polymerase (PARP), cleaved caspase- 9 and Bax. When A549 cells were treated with 0.5 or $2.0 \mu \mathrm{M}$ YH-304 for $24 \mathrm{~h}$, the expression of intact PARP was decreased and that of cleaved PARP was detected as compared with control group (Fig. 2B). Also, cleaved caspase-9 and Bax were increased by $\mathrm{YH}-304$ treatment in a dose-dependent manner (Fig. 2B). Together, these results suggest that YH-304 may induce apoptosis of A549 cells.

YH-304 does not affect p53 expression levels in HCT-116 and MDA-MB-231 cells. We previously reported that YH-304 is a simplified spiro-oxindole alkaloid which shares structural similarity with MDM2 inhibitor (an active anticancer agent) (2). Based on structural characteristics, we hypothesized that $\mathrm{YH}-304$ might show the anticancer activity through MDM2 inhibition, resulting in p53 stabilization. To investigate whether the cytotoxic effect of YH-304 is dependent on MDM2-mediated p53 modulation, we determined whether YH-304 induces p53 expression through inhibition of MDM2. We treated either YH-304 or nutlin-3 (a well-known MDM2 inhibitor) in p53 wild-type cells (HCT-116) as well as p53 mutant cells (MDA-MB-231) and then evaluated the expression levels of p53 protein and p53-dependent mRNAs including $\mathrm{p} 21$. As expected, nutlin-3 induced both $\mathrm{p} 53$ protein expression level (Fig. 3A) and p21 mRNA levels (Fig. 3B) significantly in HCT-116 cells, but not in MDA-MB-231 cells (Fig. 3C and D). However, YH-304 did not alter the mRNA or protein expression levels of p53 in the HCT-116 (Fig. 3A and E) or MDA-MB-231 cells (Fig. 3C and F). Our prelimi- 

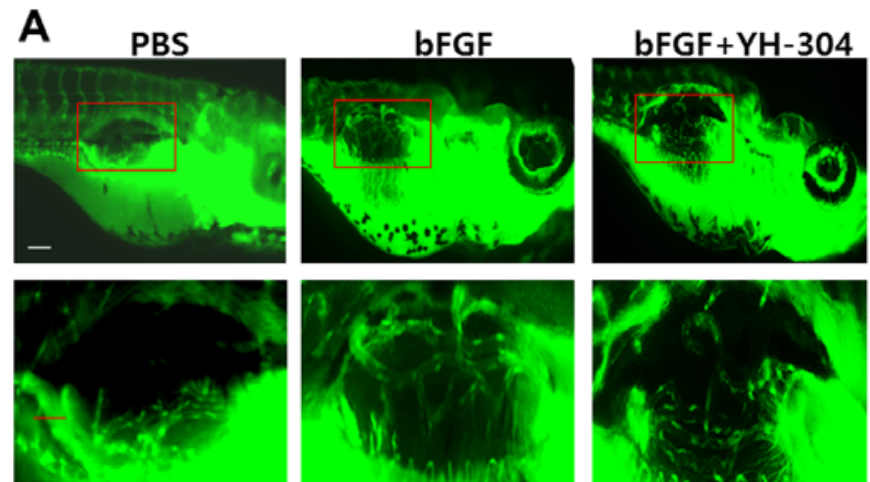

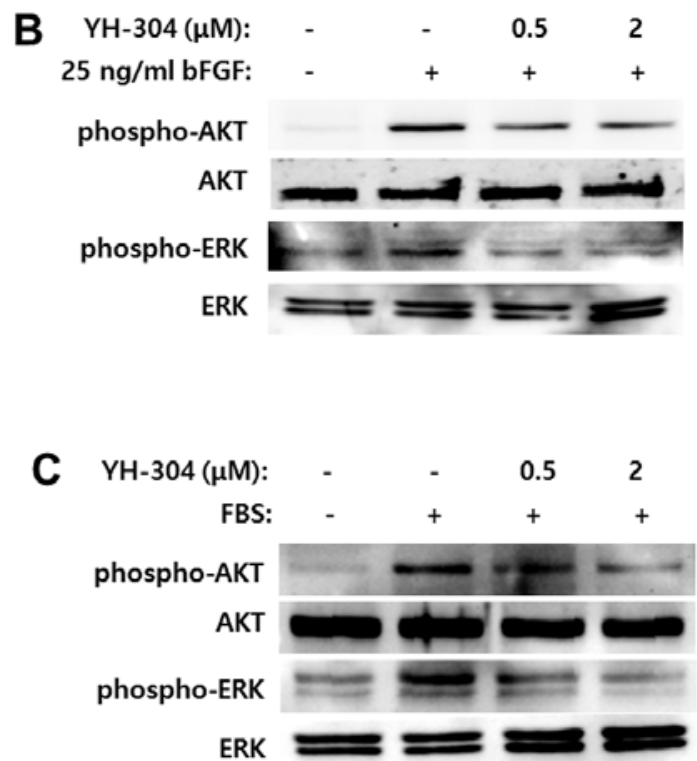

Figure 5. YH-304 inhibits bFGF-induced angiogenesis through inhibition of AKT and ERK. (A) Inhibitory effect of YH-304 in Matrigel plug assay in vivo. A total of $5 \mathrm{nl}$ of matrigel containing either PBS or bFGF $(25 \mathrm{ng} / \mathrm{ml})$ was injected into the yolk sac of each embryo of zebrafish (Danio rerio) eggs expressing green fluorescent protein (GFP) under the flk-1 promoter ( $f k-1: G F P$ ) using microinjector. YH-304 was treated immediately after Matrigel injection and embryos were examined for angiogenesis using a fluorescent microscope $24 \mathrm{~h}$ later. Scale bar is $100 \mu \mathrm{m}$. (B) Effect of YH-304 on AKT and ERK signaling mediated by recombinant bFGF. Western blots show p-AKT, total AKT, p-ERK and total ERK expression levels in A549 cells treated with YH-304 for $24 \mathrm{~h}$. Results from a representative experiment $(n=3)$ are shown. (C) Effect of YH-304 on AKT and ERK signaling mediated by $10 \%$ FBS. Western blots show p-AKT, total AKT, p-ERK and total ERK expression levels in A549 cells treated with YH-304 for $24 \mathrm{~h}$.

nary mechanistic studies of YH-304 suggested that it has anticancer activities via p53-independent pathway. Further investigations of mechanistic studies for anticancer activities are now in progress.

YH-304 shows broad spectrum anticancer activity in a MDM2-p53-independent manner. The efficacy of YH-304 was investigated in four different cancer cell lines including HCT-116 (colon cancer), HeLa (cervix cancer), A549 (lung cancer), and MDA-MB-231 (breast cancer) cells and compared with a well-known chemotherapy agent, 5-FU and nutlin-3. Compared to 5-FU, YH-304 showed potent efficacy in four different cancer cells lines, showing broad spectrum anticancer effect (Fig. 4). Especially, $\mathrm{IC}_{50}$ values of YH-304 were significantly lower than 5-FU in HCT-116, A549 and MDA-MB-231 cells (Fig. 4). Nutlin-3, a specific MDM-2 inhibitor, exerted anticancer effect in p53 wild-type cancer cells having wild-type p53 but did not affect cell viability of MDA-MB-231 cells possessing mutant p53. However, YH-304 also showed good efficacy in both p53 wild-type and p53-mutant cancer cells, suggesting that YH-304 might be a potent anticancer agent with broad-spectrum in a p53-independent fashion.

YH-304 inhibits in vivo bFGF-induced angiogenesis through suppression of AKT and ERK. To investigate the effect of YH-304 on angiogenesis in vivo, we performed matrigel plug assay with transgenic fluorescent zebrafish $\operatorname{tg}($ flil:EGFP) which expresses enhanced GFP in the entire vasculature under the control of the fli1 promoter to visualize vascular formation in live zebrafish embryos (26). In plugs with bFGF (200 ng/ml) appeared newly formed vessels (Fig. 5A). In contrast, there was less or no blood vessel formation in plugs with Matrigel alone or mixed with YH-304 (2 $\mu \mathrm{M})$ (Fig. 5A).
To further assess how YH-304 blocks bFGF-mediated angiogenesis, we tested whether $\mathrm{YH}-304$ can affect AKT and/or ERK activation, two proteins known to play an important role in the bFGF signaling pathway. As shown in Fig. 5B, bFGF increased the phosphorylation of both AKT and ERK, whereas the addition of 0.5 or $2.0 \mu \mathrm{M}$ YH-304 significantly reduced the phosphorylated AKT and phosphorylated ERK. Instead of bFGF, when treated with 10\% FBS, YH-304 showed similar inhibition of phosphorylation of both AKT and ERK (Fig. 5C). Therefore, these data suggest that YH-304 suppresses bFGF-induced angiogenesis in vivo, at least partly through blocking AKT/ERK activation.

\section{Discussion}

Cancer is now one of the leading causes of mortality worldwide, causing 7.6 million deaths in 2008 (3). Anticancer drugs are required with novel structural features to overcome multidrug resistance and severe side-effects and to enhance the effectiveness of cancer treatment $(29,30)$. Oxindole derivatives are specialized structures and exerted various biological activities, ranging from MDM2 antagonists $(31,32)$, ion channel blockers (33) and anti-inflammatory agents $(34,35)$ to various protein kinase inhibitors. A number of indole-based compounds inhibit various protein kinase families such as receptor tyrosine kinases (RTKs) and serine/ threonine-specific protein kinases $(5,10,36,37)$. As a kinase inhibitor, oxindole sunitinib was used for the treatment of advanced renal carcinoma and gastrointestinal stromal tumors $(10,11,36,37)$. We previously reported the potential use of oxindole derivatives having simplified $\alpha$-quaternary chiral lactam as anticancer agents. Among them, YH-304 had the strongest cell cytotoxicity against several cancer cell lines. 
However, the mechanisms of action underlying its anticancer effects are largely unknown and need to be better understood to allow this therapy to be used for clinical applications. This prompted us to study further the anti-tumorigenic effects of YH-304, including its underlying molecular mechanisms. Therefore, the aim of the present study was to further clarify the anticancer mechanism of $\mathrm{YH}-304$ itself, which may offer an opportunity to design effective safe drugs with minimal toxicity for the treatment of carcinoma.

The p53 tumor suppressor has been implicated as a mediator of programmed cell death (16). Loss of wild-type p53 activity is regarded as a major predictor of failure to respond to chemotherapy in various human cancers $(17,19,38)$. Therefore, patients with mutated p53 tend to be less responsive to common chemotherapeutics (39). Since p53 is the most frequently mutated in malignant cancers, therapies that do not depend on functional p53 are in general clinically preferable (39). Notably, although the structure of YH-304 is designed as a kind of oxindole derivatives similar to that of MDM2 inhibitor (a p53 activator), it did not alter the expression of p53 in p53 wild-type (HCT-116) or p53 mutant cells (MDA-MB-231), unlike nutlin-3 (MDM2 inhibitor) (Fig. 3A, C, E and F). Also, YH-304 showed similar or better efficacy in four different cancer cell lines compared to 5-FU (Fig. 4), indicating a p53-independent broad spectrum. Based on p53-independency and broad spectrum, therefore, YH-304 might be clinically preferable after further studies such as toxicity and clinical trials.

Our findings also indicate that $\mathrm{YH}-304$ interferes with AKT and ERK phosphorylation in response to angiogenic factors such as bFGF or serum. Since AKT and ERK activities are required for proliferation and tube formation of vascular endothelial cells, the AKT and ERK pathways are considered as one of the most promising targets for anti-angiogenic therapy $(40,41)$. Taken together, it is reasonable to assume that YH-304-mediated inhibition of AKT and ERK phosphorylation may be responsible for the inhibitory effect of YH-304 on angiogenesis as well as on clonogenic growth. Moreover, growing knowledge on cellular signaling networks in cancer opens up the opportunity to combine AKT and ERK inhibitors with other pathway specific inhibitors. Since synergistic effects between such inhibitors are known to exist, further studies are warranted combining YH-304 with other targeted inhibitors.

Our current data provide experimental evidence for a novel anticancer activity of YH-304 through suppression of AKT and ERK phosphorylation and, thereby, their downstream signaling cascade. Further studies are required to delineate the exact role and mode of action of YH-304, i.e., whether and how blood vessels regress in YH-304-treated Matrigel plugs. The present study provides a proof-of-principle for the development of YH-304 as an anti-angiogenic agent.

\section{Acknowledgements}

The present study was supported by the Basic Science Research Program through the National Research Foundation of Korea funded by the Ministry of Science, ICT and Future Planning (NRF-2016R1C1B2012270, NRF-2013R1A1A1059709 and NRF-2014R1A1A1002151).

\section{References}

1. Chen L, Yang J, Zheng M, Kong X, Huang T and Cai YD: The use of chemical-chemical interaction and chemical structure to identify new candidate chemicals related to lung cancer. PLoS One 10: e0128696, 2015.

2. Lee H, Hwang SJ, Jung J, Hong S, Lee M, Park HG, Lee HJ and Park Y: Asymmetric synthesis and evaluation of $\alpha$-quaternary chiral lactam derivatives as novel anticancer agents. Arch Pharm Res 37: 1264-1270, 2014.

3. Jemal A, Siegel R, Ward E, Hao Y, Xu J, Murray T and Thun MJ: Cancer statistics, 2008. CA Cancer J Clin 58: 71-96, 2008.

4. Galliford CV and Scheidt KA: Pyrrolidinyl-spirooxindole natural products as inspirations for the development of potential therapeutic agents. Angew Chem Int Ed Engl 46: 8748-8758, 2007.

5. Chen G, Weng Q, Fu L, Wang Z, Yu P, Liu Z, Li X, Zhang H and Liang G: Synthesis and biological evaluation of novel oxindolebased RTK inhibitors as anti-cancer agents. Bioorg Med Chem 22: 6953-6960, 2014.

6. Natarajan A, Guo Y, Harbinski F, Fan YH, Chen H, Luus L, Diercks J, Aktas H, Chorev M and Halperin JA: Novel arylsulfoanilide-oxindole hybrid as an anticancer agent that inhibits translation initiation. J Med Chem 47: 4979-4982, 2004.

7. Silva BV, Ribeiro NM, Vargas MD, Lanznaster M, Carneiro JW, Krogh R, Andricopulo AD, Dias LC and Pinto AC: Synthesis, electrochemical studies and anticancer activity of ferrocenyl oxindoles. Dalton Trans 39: 7338-7344, 2010.

8. Kamal A, Ramakrishna G, Raju P, Rao AV, Viswanath A, Nayak VL and Ramakrishna S: Synthesis and anticancer activity of oxindole derived imidazo[1,5-a]pyrazines. Eur J Med Chem 46: 2427-2435, 2011.

9. Cui CB, Kakeya H and Osada H: Spirotryprostatin B, a novel mammalian cell cycle inhibitor produced by Aspergillus fumigatus. J Antibiot (Tokyo) 49: 832-835, 1996.

10. Papaetis GS and Syrigos KN: Sunitinib: A multitargeted receptor tyrosine kinase inhibitor in the era of molecular cancer therapies. BioDrugs 23: 377-389, 2009.

11. Wang WL, Conley A, Reynoso D, Nolden L, Lazar AJ, George S and Trent JC: Mechanisms of resistance to imatinib and sunitinib in gastrointestinal stromal tumor. Cancer Chemother Pharmacol 67 (Suppl 1): S15-S24, 2011.

12. Kussie PH, Gorina S, Marechal V, Elenbaas B, Moreau J, Levine AJ and Pavletich NP: Structure of the MDM2 oncoprotein bound to the p53 tumor suppressor transactivation domain. Science 274: 948-953, 1996.

13. Ding K, Lu Y, Nikolovska-Coleska Z, Qiu S, Ding Y, Gao W, Stuckey J, Krajewski K, Roller PP, Tomita Y, et al: Structurebased design of potent non-peptide MDM2 inhibitors. J Am Chem Soc 127: 10130-10131, 2005.

14. Ding K, Lu Y, Nikolovska-Coleska Z, Wang G, Qiu S, ShangaryS, Gao W, Qin D, Stuckey J, Krajewski K, et al: Structure-based design of spiro-oxindoles as potent, specific small-molecule inhibitors of the MDM2-p53 interaction. J Med Chem 49: 3432-3435, 2006

15. Park Y, Lee YJ, Hong S, Lee M and Park HG: Highly enantioselective total synthesis of (+)-isonitramine. Org Lett 14: 852-854, 2012.

16. Reed SM and Quelle DE: p53 Acetylation: Regulation and consequences. Cancers (Basel) 7: 30-69, 2014.

17. Melvin AT, Dumberger LD, Woss GS, Waters ML and Allbritton NL: Identification of a p53-based portable degron based on the MDM2-p53 binding region. Analyst (Lond) 141: 570-578, 2016.

18. Huang KY, Weng JT, Lee TY and Weng SL: A new scheme to discover functional associations and regulatory networks of E3 ubiquitin ligases. BMC Syst Biol 10 (Suppl 1): 3, 2016.

19. Richmond J, Carol H, Evans K, High L, Mendomo A, Robbins A, Meyer C, Venn NC, Marschalek R, Henderson M, et al: Effective targeting of the P53-MDM2 axis in preclinical models of infant MLL-rearranged acute lymphoblastic leukemia. Clin Cancer Res 21: 1395-1405, 2015

20. Saiki AY, Caenepeel S, Cosgrove E, Su C, Boedigheimer M and Oliner JD: Identifying the determinants of response to MDM2 inhibition. Oncotarget 6: 7701-7712, 2015.

21. Deben C, Wouters A, Op de Beeck K, van Den Bossche J, Jacobs J, Zwaenepoel K, Peeters M, Van Meerbeeck J, Lardon F, Rolfo C, et al: The MDM2-inhibitor Nutlin-3 synergizes with cisplatin to induce 533 dependent tumor cell apoptosis in non-small cell lung cancer. Oncotarget 6: 22666-22679, 2015. 
22. Zanjirband M, Edmondson RJ and Lunec J: Pre-clinical efficacy and synergistic potential of the MDM2-p53 antagonists, Nutlin-3 and RG7388, as single agents and in combined treatment with cisplatin in ovarian cancer. Oncotarget: May 20, 2016 (Epub ahead of print). doi: 10.18632/oncotarget.9499.

23. Michaelis M, Rothweiler F, Barth S, Cinatl J, van Rikxoort M, Löschmann N, Voges Y, Breitling R, von Deimling A, Rödel F, et al: Adaptation of cancer cells from different entities to the MDM2 inhibitor nutlin-3 results in the emergence of p53-mutated multi-drug-resistant cancer cells. Cell Death Dis 2: e243, 2011.

24. Rennekamp AJ and Peterson RT: 15 years of zebrafish chemical screening. Curr Opin Chem Biol 24: 58-70, 2015.

25. Sun Y, Sheng Z, Ma C, Tang K, Zhu R, Wu Z, Shen R, Feng J, Wu D, Huang D, et al: Combining genomic and network characteristics for extended capability in predicting synergistic drugs for cancer. Nat Commun 6: 8481, 2015.

26. Liu $\mathrm{Z}$ and Liu F: Cautious use of flila:EGFP transgenic zebrafish in vascular research. Biochem Biophys Res Commun 427: 223-226, 2012.

27. Barriuso J, Nagaraju R and Hurlstone A: Zebrafish: A new companion for translational research in oncology. Clin Cancer Res 21: 969-975, 2015.

28. Eden CJ, Ju B, Murugesan M, Phoenix TN, Nimmervoll B, Tong Y, Ellison DW, Finkelstein D, Wright K, Boulos N, et al: Orthotopic models of pediatric brain tumors in zebrafish. Oncogene 34: 1736-1742, 2015.

29. Gonçalves AS, Macedo AS and Souto EB: Therapeutic nanosystems for oncology nanomedicine. Clin Transl Oncol 14: 883-890, 2012.

30. Yin Q, Shen J, Zhang Z, Yu H, Chen L, Gu W and Li Y: Multifunctional nanoparticles improve therapeutic effect for breast cancer by simultaneously antagonizing multiple mechanisms of multidrug resistance. Biomacromolecules 14: 2242-2252, 2013.

31. Ribeiro CJ, Amaral JD, Rodrigues CM, Moreira R and Santos MM: Synthesis and evaluation of spiroisoxazoline oxindoles as anticancer agents. Bioorg Med Chem 22: 577-584, 2014.
32. Shangary S, Ding K, Qiu S, Nikolovska-Coleska Z, Bauer JA, Liu M, Wang G, Lu Y, McEachern D, Bernard D, et al: Reactivation of p53 by a specific MDM2 antagonist (MI-43) leads to p21-mediated cell cycle arrest and selective cell death in colon cancer. Mol Cancer Ther 7: 1533-1542, 2008.

33. Jensen BS: BMS-204352: A potassium channel opener developed for the treatment of stroke. CNS Drug Rev 8: 353-360, 2002.

34. Chen G, Jiang L, Dong L, Wang Z, Xu F, Ding T, Fu L, Fang Q, Liu Z, Shan X, et al: Synthesis and biological evaluation of novel indole-2-one and 7-aza-2-oxindole derivatives as anti-inflammatory agents. Drug Des Devel Ther 8: 1869-1892, 2014.

35. Sun Y, Liu J, Jiang X, Sun T, Liu L, Zhang X, Ding S, Li J, Zhuang Y, Wang Y, et al: One-step synthesis of chiral oxindoletype analogues with potent anti-inflammatory and analgesic activities. Sci Rep 5: 13699, 2015.

36. Ho HK, Chua BT, Wong W, Lim KS, Teo V, Ong HT, Chen X, Zhang W, Hui KM, Go ML, et al: Benzylidene-indolinones are effective as multi-targeted kinase inhibitor therapeutics against hepatocellular carcinoma. Mol Oncol 8: 1266-1277, 2014.

37. Izzedine H, Buhaescu I, Rixe O and Deray G: Sunitinib malate. Cancer Chemother Pharmacol 60: 357-364, 2007.

38. Engeland K: Simplify p53: Just an activator. Oncotarget 6: 3-4, 2015.

39. Koshino A, Goto-Koshino Y, Setoguchi A, Ohno K and Tsujimoto H: Mutation of $\mathrm{p} 53$ gene and its correlation with the clinical outcome in dogs with lymphoma. J Vet Intern Med 30: 223-229, 2016.

40. Hellesøy M and Lorens JB: Cellular context-mediated Akt dynamics regulates MAP kinase signaling thresholds during angiogenesis. Mol Biol Cell 26: 2698-2711, 2015.

41. Huang JJ, Shi YQ, Li RL, Hu A, Lu ZY, Weng L, Wang SQ, Han YP, Zhang L, Li B, et al: Angiogenesis effect of therapeutic ultrasound on HUVECs through activation of the PI3K-AkteNOS signal pathway. Am J Transl Res 7: 1106-1115, 2015. 